Deformation method for generalized Abelian Higgs-Chern-Simons models

This content has been downloaded from IOPscience. Please scroll down to see the full text. 2013 EPL 10131001

(http://iopscience.iop.org/0295-5075/101/3/31001)

View the table of contents for this issue, or go to the journal homepage for more

Download details:

IP Address: 200.130.19.138

This content was downloaded on 06/03/2015 at $14: 15$

Please note that terms and conditions apply. 


\title{
Deformation method for generalized Abelian Higgs-Chern-Simons models
}

\author{
L. Losano ${ }^{1,2,3}$, J. M. C. Malbouisson ${ }^{1,4}$, D. Rubiera-Garcia ${ }^{5}$ and C. dos Santos $^{1}$ \\ ${ }^{1}$ Centro de Fúsica e Departamento de Fúsica e Astronomia, Faculdade de Ciências da Universidade do Porto \\ 4169-007 Porto, Portugal, EU \\ ${ }^{2}$ Departamento de Física, Universidade Federal da Paraíba - 58051-900 João Pessoa, PB, Brazil \\ ${ }^{3}$ Departamento de Física, Universidade Federal de Campina Grande - 58109-970 Campina Grande, PB, Brazil \\ ${ }^{4}$ Instituto de Física, Universidade Federal da Bahia - 40210-340 Salvador, BA, Brazil \\ ${ }^{5}$ Departamento de Física, Universidad de Oviedo - 33007 Oviedo, Asturias, Spain, EU
}

received 30 December 2012; accepted 25 January 2013

published online 11 February 2013

PACS 11.27.+d - Extended classical solutions; cosmic strings, domain walls, texture PACS 11.15. Yc - Chern-Simons gauge theory

\begin{abstract}
We present an extension of the deformation method applied to self-dual solutions of generalized Abelian Higgs-Chern-Simons models. Starting from a model defined by a potential $V(|\phi|)$ and a non-canonical kinetic term $\omega(|\phi|)\left|D_{\mu} \phi\right|^{2}$ whose analytical domain-wall solutions are known, we show that this method allows to obtain infinitely many new analytic solutions of new models defined by other functions $\widetilde{V}$ and $\widetilde{\omega}$. We present some examples of deformation functions leading to new families of models and their associated analytic solutions.
\end{abstract}

Copyright (C) EPLA, 2013

Introduction. - Topological defects play an important role in several areas of modern theoretical physics, such as high-energy physics [1], cosmology [2] and condensedmatter physics [3]. Such defects emerge as classical solutions of nonlinear field theories which possess degenerated vacua. Typical examples are domain walls described by kink solutions of the $\phi^{4}$ model, Ginzburg-Landau vortices and monopoles.

Usually, domain walls are solutions connecting two distinct vacua of scalar field theories in one-space dimension, or in their embedding in higher dimensions, while vortices emerge as solutions of models that couple charged matter fields with gauge fields living in a (at least) 3-dimensional space-time, and monopoles in four spacetime dimensions.

In a $(2+1)$-dimensional space-time, minimal coupling between charged matter and gauge fields can be implemented by the Chern-Simons (CS) action. Although the CS field cannot be conceived as a free field, its coupling with matter fields imposes constraints in the dynamics which have very relevant consequences, both in classical and quantum theories, with either relativistic or non-relativistic kinetics. In the non-relativistic (NR) framework, particles coupled through the CS field carry both electric charge and magnetic flux, and possess fractional statistics [4]. Additionally, the NR scalar CS model constitutes a seminal example of a Galilean-invariant gauge-field theory [5]. Also, for a critical strength of a quartic self-interaction of the scalar field, which restores the scale invariance [6], this model provides a field-theoretical description of the Aharonov-Bohm (AB) scattering [7]; considering the Lorentz covariant field theory, relativistic corrections to the $\mathrm{AB}$ scattering have been obtained [8].

Self-dual soliton solutions have been found in the relativistic, $U(1)$-invariant, Abelian, Higgs-Chern-Simons (HCS) gauge theory where the symmetry-breaking potential of the Higgs field is $U(\varphi) \sim|\varphi|^{2}\left(|\varphi|^{2}-v^{2}\right)^{2}[9]$; vortex and domain-wall solutions have been also obtained [10]. This model was generalized by considering a non-canonical kinetic term for the complex scalar field, $\mathcal{W}(|\varphi|)\left|D_{\mu} \varphi\right|^{2}$, providing self-dual vortex [11] and domain-wall [12] solutions. Models with non-canonical kinetic terms ( $k$-fields) find also applications in strong-interaction physics [13] and in cosmology [14].

Due to the nonlinearity, there is no general integration method to solve analytically the equations of motion of nonlinear field theories; only for a small set of models, solutions of the equations of motion can be directly determined. However, for scalar fields in $(1+1)$-dimensions, starting from a nonlinear model with known solutions, infinitely many new models and their corresponding static 
solutions can be found using the deformation method [15]. This method works as follows. Choosing a deformation function $f(\phi)$, the model defined by the deformed potential $\widetilde{V}(\phi)=V[(f(\phi))] /\left[f^{\prime}(\phi)\right]^{2}$, where $f^{\prime}$ means the derivative of $f$, possesses static solutions given by $\widetilde{\phi}(x)=$ $f^{-1}(\phi(x))$, where $\phi(x)$ is a solution of the static equation of motion of the original model with potential $V(\phi)$. This procedure has been applied to generate defect solutions of many models having polynomial interactions [16] and new families of sine-Gordon and multi-sine-Gordon models [17]. Also, an orbit-based extension of this method has been applied to models involving two interacting scalar fields [18].

The purpose of this letter is to extend the deformation method to gauge field models considering specifically the Abelian HCS theory, focusing particularly on the Jackiw-Lee-Weinberg (JLK) domain-wall solution [10]. Firstly, we present the generalized Abelian HCS models and write down the first-order equations obeyed by the Bogomol'nyi-Prasad-Sommerfeld (BPS) [19] domain-wall solutions. Then, the deformation method is extended to domain-wall solutions of generalized Abelian HCS models and some examples are given, illustrating the power of the procedure in generating new models with their static solutions. Finally, some remarks are made.

BPS domain walls in the generalized Abelian HCS model. - We consider the generalized $(2+1)$ dimensional Abelian HCS model defined by the Lagrangian density [11]

$$
\mathcal{L}_{\mathcal{S}}=\mathcal{W}(|\varphi|)\left|D_{\mu} \varphi\right|^{2}-U(|\varphi|)+\frac{\kappa}{4} \epsilon^{\alpha \beta \gamma} \mathcal{A}_{\alpha} \mathcal{F}_{\beta \gamma},
$$

where $\varphi$ is the complex Higgs field, $D_{\mu}=\partial_{\mu}+i e \mathcal{A}_{\mu}$ is the covariant derivative and $\mathcal{F}_{\mu \nu}=\partial_{\mu} \mathcal{A}_{\nu}-\partial_{\nu} \mathcal{A}_{\mu}$ is the field strength tensor of the gauge potential $\mathcal{A}_{\mu}$. The selfinteraction potential, $U(|\varphi|)$, is assumed to implement a symmetry-breaking mechanism and the non-canonicity of the kinetic term is engendered by the function $\mathcal{W}(|\varphi|)$; taking $\mathcal{W} \equiv 1$, one recovers the standard Abelian HCS model. Note that, in the CS term, $\epsilon^{\alpha \beta \gamma}$ is the fully antisymmetric tensor and the electric and the magnetic CS fields are $\mathcal{E}^{i}=\mathcal{F}^{i 0}=-\partial_{0} \mathcal{A}^{i}-\nabla_{i} \mathcal{A}^{0}$ and $\mathcal{B}=\vec{\nabla} \times \overrightarrow{\mathcal{A}}=$ $\partial_{2} \mathcal{A}_{1}-\partial_{1} \mathcal{A}_{2}$, respectively.

It is convenient to work with dimensionless quantities. In $(2+1)$ dimensions, the scalar field $\varphi$ has mass dimension equal to $1 / 2$, the same that we take for the gauge field; this choice ensures that the mass dimension of $\mathcal{A}_{\alpha}$ agrees with the one obtained if a Maxwell term were added to $\mathcal{L}_{\mathcal{S}}$. It follows that the electric charge $e$ and the CS parameter $\kappa$ have mass dimensions equal to $1 / 2$ and 1 , respectively, so that $e^{2} / \kappa$ is dimensionless. We can get an additional simplification if we absorb the parameters $e$ and $\kappa$ by redefining space-time coordinates and fields. Thus, with $M$ being a mass scale of the model, we define $\bar{x}^{\mu}=M e^{2} x^{\mu} / \kappa, \phi=\sqrt{\kappa} \varphi / \sqrt{M} e, A_{\mu}=$ $\kappa \mathcal{A}_{\mu} / M e, V=\kappa^{2} U / M^{3} e^{4}$ and $\omega=e^{2} \mathcal{W} / \kappa$; the dimensionless Lagrangian density is then given by $\mathcal{L}=\kappa^{2} \mathcal{L}_{\mathcal{S}} / M^{3} e^{4}$ and the action becomes $\mathcal{S}=\frac{\kappa}{e^{2}} \int \mathrm{d}^{3} \bar{x} \mathcal{L}$. To simplify the notation, we remove the bar over the space-time coordinates and use, from now on, only dimensionless quantities.

Variation of the action leads to the equations of motion

$$
\begin{gathered}
\omega D_{\mu} D^{\mu} \phi+\partial_{\mu} \omega D^{\mu} \phi-\left|D_{\mu} \phi\right|^{2} \frac{\partial \omega}{\partial \phi^{*}}+\frac{\partial V}{\partial \phi^{*}}=0 \\
\frac{1}{2} \epsilon^{\alpha \beta \gamma} F_{\beta \gamma}=-J^{\alpha}
\end{gathered}
$$

where the current density, $J^{\alpha}=(\rho, \vec{j})$, is given by

$$
J^{\alpha}=i \omega\left[\phi\left(D^{\alpha} \phi\right)^{*}-\phi^{*} D^{\alpha} \phi\right] .
$$

The time component of eq. (3) states that the magnetic field is equal to the planar electric-charge density, $B=\rho$, which is the CS Gauss law. Also, for static field configurations, we find

$$
B=\rho=2 A_{0}|\phi|^{2} \omega(|\phi|), \quad E^{a}=\epsilon_{a b} j^{b},
$$

which shows that the electric-current density is perpendicular to the electric field.

The energy-momentum tensor is given by

$$
\begin{aligned}
T_{\mu \nu}= & \omega\left[D_{\mu} \phi\left(D_{\nu} \phi\right)^{*}+D_{\nu} \phi\left(D_{\mu} \phi\right)^{*}\right] \\
& -g_{\mu \nu}\left[\omega\left|D_{\alpha} \phi\right|^{2}-V(|\phi|)\right]
\end{aligned}
$$

from which we obtain the energy density, $\varepsilon=T_{00}$, and the pressure components, $\mathcal{P}_{1}=T_{11}$ and $\mathcal{P}_{2}=T_{22}$.

We are interested in static domain-wall solutions. Firstly, note that the complex phase of the scalar field $\phi$ can be suppressed by a suitable gauge transformation. Then, fixing the Coulomb gauge, we can search for solutions of the form $[10,12]$

$$
\phi=h(x), \quad A_{\mu}=\left(A_{0}(x), A_{1}=0, A_{2}=A(x)\right),
$$

where $h(x)$ and $A(x)$ are real functions and $x$ denotes the $x^{1}$-coordinate. This ansatz corresponds to domain walls (actually lines in the plane) parallel to the $x^{2}$-axis.

In this case, the static equations of motion reduce to

$$
\begin{aligned}
{\left[2 \omega h^{\prime}\right]^{\prime} } & =2 h \omega\left(A^{2}-A_{0}^{2}\right)+\frac{\mathrm{d} V}{\mathrm{~d} h}, \\
A_{0}^{\prime} & =-2 \omega h^{2} A
\end{aligned}
$$

and the Gauss law

$$
A^{\prime}=-2 \omega h^{2} A_{0}
$$

where the prime denotes derivation with respect to $x$. From eqs. (9) and (10) we infer that $A_{0} A_{0}^{\prime}=A A^{\prime}$, so that time and space components of the gauge field are constrained by

$$
A_{0}^{2}=A^{2}-C,
$$

where $C$ is a real constant. Also, consistency with eq. (8) imposes a relation between the function $\omega(h)$ and the potential $V(h)$ expressed as

$$
\frac{\mathrm{d}}{\mathrm{d} h}\left[\frac{\sqrt{V / \omega}}{h}\right]=-2 \omega h .
$$


Now, the stability condition $\mathcal{P}_{1}=\mathcal{P}_{2}=0$ leads to the first-order equations [20]

$$
\begin{aligned}
& h^{\prime}= \pm h A, \\
& A^{\prime}=-2 \omega h^{2} A_{0},
\end{aligned}
$$

with

$$
V=h^{2} \omega A_{0}^{2}
$$

For $h \geqslant 0$ and $A \geqslant 0$, the sign $+(-)$ in eq. (13) corresponds to the kink (anti-kink) like solution for the Higgs field, $h^{(+)}$ $\left(h^{(-)}\right)$. Note that the first-order equations (13) and (14) solve the equations of motion (2) and (3).

The static solutions are physically characterized by their charge and energy. Now, returning to eq. (6), for nonnegative $V(h)$ and $\omega(h)$, the energy of static solutions can be rewritten under the form

$$
\begin{aligned}
E= & \int_{-\infty}^{\infty} \mathrm{d} x T_{00} \\
= & \int_{-\infty}^{\infty} \mathrm{d} x\left(V+\omega h^{\prime}+2 \omega h^{2} A_{0}^{2}+C \omega h^{2}\right) \\
= & \int_{-\infty}^{\infty} \mathrm{d} x\left[\left(\sqrt{V} \pm \sqrt{\omega} h A_{0}^{2}\right)^{2}+\left(\sqrt{\omega} h^{\prime} \pm \sqrt{\omega} h A\right)^{2}\right. \\
& \left.+\left(\sqrt{-A_{0} A^{\prime}} \pm \sqrt{2 \omega} h A_{0}\right)^{2}\right] \\
& +\int_{-\infty}^{\infty} \mathrm{d} x\left(2 \sqrt{\omega V} h A_{0} \pm 2 \omega h h^{\prime} A\right. \\
& \left. \pm 2 \sqrt{-2 \omega A_{0} A^{\prime}} h A_{0}+A_{0} A^{\prime}-2 \omega h^{2} A_{0}^{2}\right)
\end{aligned}
$$

which is minimized if eqs. (13), (14), and (15) are obeyed, resulting in (for $C=0$ )

$$
E=\int_{-\infty}^{\infty} \mathrm{d} x(4 V)=\left|\left(A^{2}(-\infty)-A^{2}(+\infty)\right)\right| .
$$

In this case $A_{0}^{2}=A^{2}$, so the system of first-order equations decouples and is solved simply by (13) with

$$
A(h)=-2 \int \omega h \mathrm{~d} h+c,
$$

where $c$ is an integration constant suitable to the boundary conditions required for the gauge field. And, from (5) and (6), the electric charge, $Q$, and Noether charge, $P$, are given by

$$
\begin{aligned}
& Q=\int_{-\infty}^{\infty} \mathrm{d} x \rho(x)=A(-\infty)-A(+\infty), \\
& P=\int_{-\infty}^{\infty} \mathrm{d} x T_{02}=\frac{1}{2}\left[A^{2}(-\infty)-A^{2}(+\infty)\right],
\end{aligned}
$$

and both are conserved due to the $U(1)$ symmetry and the translational invariance along the $x^{2}$-direction, respectively.

This shows that, for $h$ in a range such that $\omega(h) \geqslant 0$ and $V(h) \geqslant 0$, the BPS solutions of the first-order equations (13) and (14) (together with (15)), indeed correspond to solutions of minimum energy and their energy

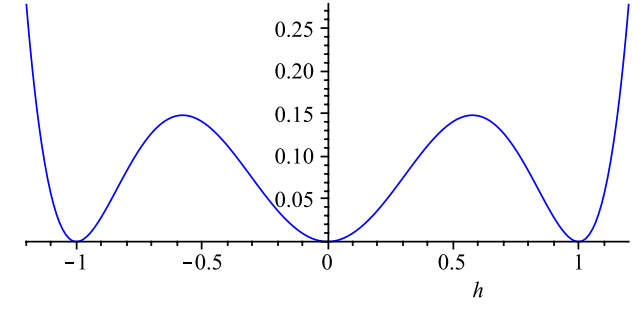

Fig. 1: (Colour on-line) The potential (21) as a function of the Higgs field.
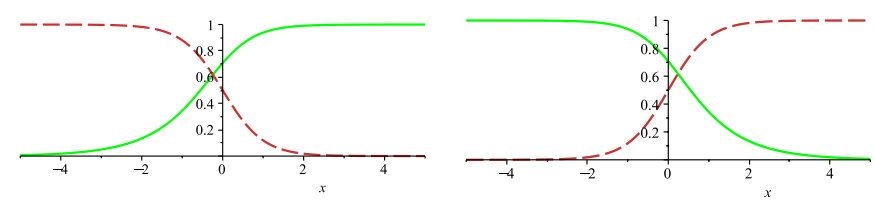

Fig. 2: (Colour on-line) The Higgs field (solid line) and the gauge field (dashed line), $\left(h^{(+)}(x), A^{(-)}(x)\right)$ from eq. (23) on the left, and $\left(h^{(-)}(x), A^{(+)}(x)\right)$ from eq. (24) on the right.

and charge can be calculated knowing only the asymptotic behavior of the gauge field. Correspondingly, the Higgs field, for both kink and anti-kink solutions, connects two consecutive vacua of the potential, while a lump-like solution starts and terminates on the same vacuum when $x \rightarrow \pm \infty$.

Standard self-dual domain walls. The simplest Abelian HCS model that supports self-dual domain-wall solutions is the JLW model [10], which is defined by the Lagrangian (1) with canonical kinetic term $(\omega=1)$ and the (dimensionless) symmetry-breaking potential

$$
V(h)=h^{2}\left(1-h^{2}\right)^{2},
$$

plotted in fig. 1. In this case, the use of eq. (18) (with $c=1$ ) provides the result

$$
A=1-h^{2}
$$

which, substituting in (13), gives the pair of solutions

$$
h^{(+)}(x)=1 / \sqrt{1+e^{-2 x}}, A^{(-)}(x)=1 /\left(1+e^{2 x}\right),
$$

and

$$
h^{(-)}(x)=1 / \sqrt{1+e^{2 x}}, A^{(+)}(x)=1 /\left(1+e^{-2 x}\right),
$$

which are displayed in fig. 2. We see that the scalar field, in both cases, interpolates between the symmetric and the asymmetric vacua. Figure 3 shows the energy and electric-charge densities for both wall solutions. We find that the spatial distribution of the electric charge is symmetric around the origin, while for the energy the axis of symmetry is displaced from the origin. And from eqs. (17), (19) and (20), for the solutions $\left(h^{(+)}, A^{(-)}\right)$ and $\left(h^{(-)}, A^{(+)}\right)$, we have the charges $Q=1, P=1 / 2$, and $Q=-1, P=-1 / 2$, respectively, and the same energy, $E=1$. 


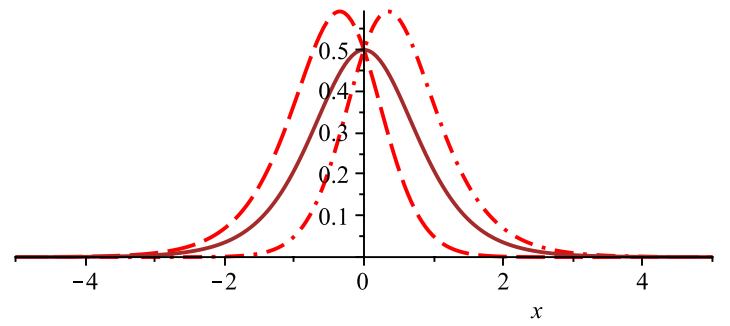

Fig. 3: (Colour on-line) Energy density of the solutions $\left(h^{(+)}(x), A^{(-)}(x)\right)$ (dashed line) and $\left(h^{(-)}(x), A^{(+)}(x)\right)$ (dashdotted line), and module of electric-charge density for both solutions (solid line).

The deformation method. - Let us now develop the deformation method for generalized Abelian HCS models following the spirit of the procedure introduced for scalar fields [15]. As we shall show, by deforming simultaneously the Higgs and the CS fields, we are able to construct many new generalized HCS models and their static analytic domain-wall solutions. The original and the deformed models are mapped into each other through the deformation function.

Denote by $\tilde{\phi}(x)$ and $\widetilde{A}(x)$ new fields whose dynamics is governed by the (dimensionless) Lagrangian density

$$
\widetilde{\mathcal{L}}=\widetilde{\omega}(|\tilde{\phi}|)\left|D_{\mu} \tilde{\phi}\right|^{2}-\tilde{V}(|\tilde{\phi}|)+\frac{1}{4} \epsilon^{\alpha \beta \gamma} \widetilde{A}_{\alpha} \widetilde{F}_{\beta \gamma}
$$

where $\widetilde{V}(|\tilde{\phi}|)$ and $\widetilde{\omega}(|\tilde{\phi}|)$ are new functions specifying this model. As we did before, we assume that the self-dual BPS domain-wall solutions of this model take the form

$$
\tilde{\phi}=\tilde{h}(x), \quad \widetilde{A}_{\mu}=\left(\widetilde{A}_{0}(x), \widetilde{A}_{1}=0, \widetilde{A}_{2}=\widetilde{A}(x)\right),
$$

and satisfy the first-order equations of motion

$$
\begin{aligned}
& \tilde{h}^{\prime}= \pm \tilde{h} \widetilde{A}, \\
& \widetilde{A}^{\prime}=-2 \widetilde{\omega} \tilde{h}^{2} \widetilde{A}_{0},
\end{aligned}
$$

where $\tilde{h}^{\prime} \equiv \mathrm{d} \tilde{h} / \mathrm{d} x$ and $\widetilde{A}^{\prime} \equiv \mathrm{d} \widetilde{A} / \mathrm{d} x$, with the constraints $\widetilde{V}=h^{2} \widetilde{\omega} \widetilde{A}_{0}^{2}$ and $\widetilde{A}_{0}^{2}=\widetilde{A}^{2}$.

Now, introduce the deformation function $f$ such that the Higgs fields of the two models are mapped into each other, $h=f(\tilde{h})$, which is assumed to be invertible (in a prescribed domain of definition) and differentiable. Also, consider that the deformed CS-gauge field is obtained from $A$ by the prescription

$$
\widetilde{A}(\tilde{h})=\frac{f(\tilde{h}) A[h \rightarrow f(\tilde{h})]}{\tilde{h} f_{\widetilde{h}}},
$$

where $f_{\widetilde{h}}=\mathrm{d} f / \mathrm{d} \widetilde{h}$. Then, it follows from eqs. (27) and (28), using eq. (29), that the model defined by the Lagrangian density (25), with the deformed function $\widetilde{\omega}$ and the deformed potential $\widetilde{V}$ given by

$$
\widetilde{\omega}(\tilde{h})=\frac{1}{2} \frac{\widetilde{A}_{\tilde{h}}}{\tilde{h}}, \quad \tilde{V}(\tilde{h})=\tilde{h}^{2} \widetilde{A}^{2} \widetilde{\omega}(\tilde{h}),
$$
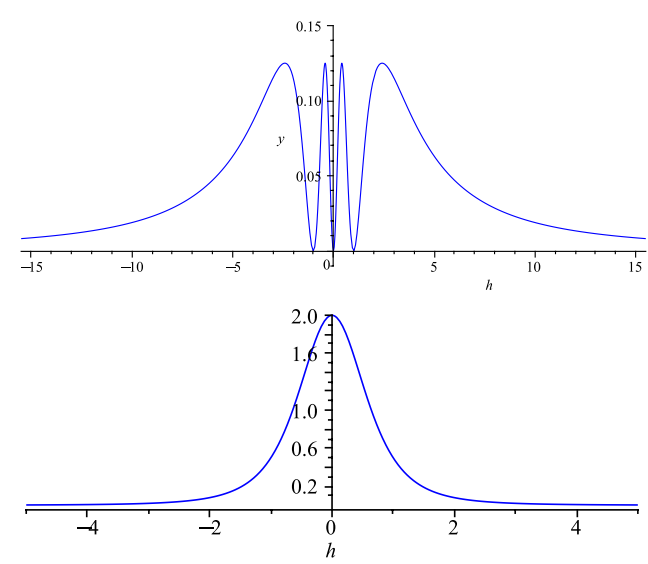

Fig. 4: (Colour on-line) The potential (33) (top panel) and the corresponding function $w$ (bottom panel), as a function of the Higgs field.

where $\widetilde{A}_{\tilde{h}}=\mathrm{d} \tilde{A} / \mathrm{d} \tilde{h}$, possesses static BPS solutions

$$
\tilde{h}(x)=f^{-1}[h(x)], \quad \widetilde{A}(x)=\widetilde{A}\left(f^{-1}[h(x)]\right),
$$

where $h(x)$ is a static solution of the original model (1).

It should be noted that all the considerations and relations presented before, relative to energy and conserved charges, are held unchanged for the deformed system. In the following, taking as the starting point the JLW domain-wall solutions, we consider some illustrative examples of the method.

Example I. Firstly, we consider the pair of deformation functions

$$
f(\tilde{h})^{( \pm)}=( \pm) \frac{1-\tilde{h}^{2}}{1+\tilde{h}^{2}}
$$

which, using eqs. (22) and $(29)$, gives $\widetilde{A}^{( \pm)}(\tilde{h})=f(\tilde{h})^{( \pm)}$; and, from eq. (30), it follows that

$$
\tilde{\omega}=\frac{2}{\left(1+\tilde{h}^{2}\right)^{2}}, \quad \tilde{V}=\frac{2 \tilde{h}^{2}\left(1-\tilde{h}^{2}\right)^{2}}{\left(1+\tilde{h}^{2}\right)^{4}} .
$$

These functions, plotted in fig. 4, define the generalized Abelian HCS model employed in ref. [12]. Note that the three vacua at $\tilde{h}=0,1,+\infty$ establish two walls, one between $\tilde{h}=0$ and $\tilde{h}=1$, and the other between $\tilde{h}=1$ and $\tilde{h}=+\infty$. From the inverse of the deformation function (32) and eqs. (23) and (24), for the range $0 \leqslant \tilde{h} \leqslant 1$, we obtain the solutions

$\tilde{h}^{(+)}(x)=\sqrt{1+e^{-2 x}}-e^{-x}, \quad \widetilde{A}^{(-)}(x)=1 / \sqrt{1+e^{2 x}}$,

$$
\tilde{h}^{(-)}(x)=1 / \sqrt{1+2 e^{2 x}}, \quad \widetilde{A}^{(+)}(x)=1 /\left(1+e^{-2 x}\right),
$$

while for $\tilde{h} \geqslant 1$ we have

$\tilde{h}^{(+)}(x)=\sqrt{1+2 e^{2 x}}, \quad \widetilde{A}^{(+)}(x)=1 /\left(1+e^{-2 x}\right)$, 

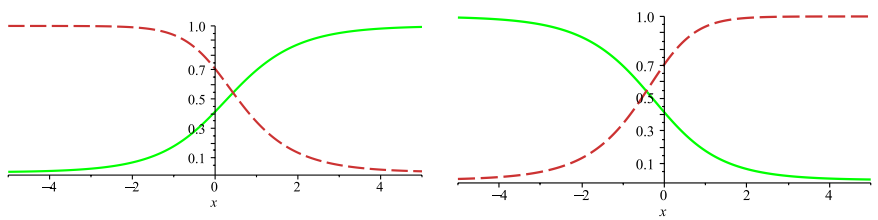

Fig. 5: (Colour on-line) The Higgs field (solid line) and the gauge field (dashed line), $\left(\tilde{h}^{(+)}(x), \widetilde{A}^{(-)}(x)\right)$ from eq. (34) on the left, and $\left(\tilde{h}^{(-)}(x), \widetilde{A}^{(+)}(x)\right)$ from eq. (35) on the right, for $0 \leqslant \tilde{h} \leqslant 1$.
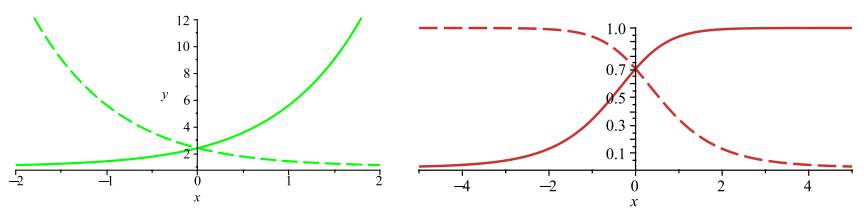

Fig. 6: (Colour on-line) The fields $\left(\tilde{h}^{(+)}(x), \widetilde{A}^{(+)}(x)\right)$, eq. (36) (solid line), and $\left(\tilde{h}^{(-)}(x), \widetilde{A}^{(-)}(x)\right)$, eq. (37) (dashed line), for $\tilde{h} \geqslant 1$.

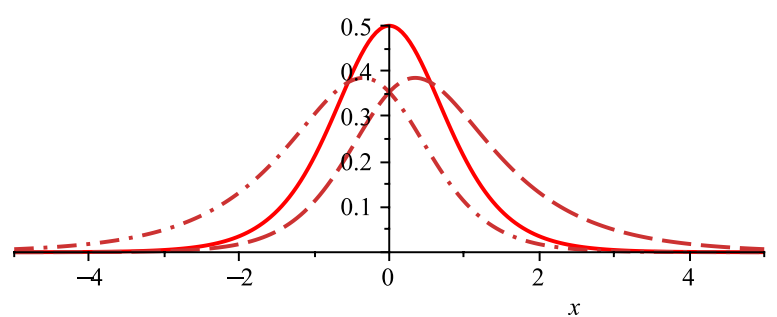

Fig. 7: (Colour on-line) Module of electric charge for solutions $A^{(-)}(x)$ (dashed line) and $A^{(+)}(x)$ (dash-dotted line), and energy density (solid line) for both walls.

$$
\tilde{h}^{(-)}(x)=\sqrt{1+e^{-2 x}}+e^{-x}, \quad \widetilde{A}^{(-)}(x)=1 /\left(\sqrt{1+e^{2 x}}\right) .
$$

In figs. 5 and 6 we display these domain-wall solutions. The walls for $0 \leqslant \tilde{h} \leqslant 1$ and $\tilde{h} \geqslant 1$ have the same gauge fields, but with the asymptotic value for $x= \pm \infty$ changed. Then, for both ranges the walls have the same energy, $E=1$, and charges $Q=1$ and $P=1 / 2$, for $\widetilde{A}^{(-)}$, and $Q=-1$ and $P=-1 / 2$, for $\widetilde{A}^{(+)}$. This makes to have attractive or repulsive force between the two walls possible.

In fig. 7, we display the energy and charge densities for the two walls. The comparison with the walls of the JLW model shows that, notwithstanding the walls have the same charges and energy, the JLW walls have symmetric spatial distributions of energy and charge, while here only the distribution of energy is symmetric and all the corresponding distributions are more spread out. The model defined by eqs. (33), which was obtained by deforming the JLW model, was studied in ref. [12] but only the solution satisfying $0 \leqslant \tilde{h} \leqslant 1$ was considered.

Let us note that (32) is a particular case of the deformation function $f(\tilde{h})=\cos [\alpha \arctan (\tilde{h})]$, corresponding to $\alpha=2$; from that function a new family of models can be generated for $\alpha$ integer.

Example II. As a second example, consider the set of deformation functions [16]

$$
f_{\alpha}(\tilde{h})=\cos [\alpha \arccos (\tilde{h})]=T_{\alpha}(\tilde{h}),
$$

where the integer $\alpha>2$ and $T_{\alpha}$ are the Chebyshew polynomials of first kind. Using this deformation in eq. (29) and taking into account eq. (22), we have the gauge field

$$
\begin{aligned}
\widetilde{A}_{\alpha}(\tilde{h}) & =\left(1-\tilde{h}^{2}\right)^{1 / 2} \sin [2 \alpha \arccos (\tilde{h})] / 2 \alpha \tilde{h} \\
& =\left(1-\tilde{h}^{2}\right) U_{2 \alpha-1}(\tilde{h}) / 2 \alpha \tilde{h},
\end{aligned}
$$

where $U_{\sigma}$ are the Chebyshew polynomials of second kind. Explicitly, for $\alpha=2,3$, eq. (39) reads

$$
\begin{aligned}
& \widetilde{A}_{2}(\tilde{h})=\left(1-\tilde{h}^{2}\right)\left(2 \tilde{h}^{2}-1\right), \\
& \widetilde{A}_{3}(\tilde{h})=\left(1-\tilde{h}^{2}\right)\left(1-2 \tilde{h}^{2}\right)\left(3-4 \tilde{h}^{2}\right) / 3 .
\end{aligned}
$$

In this case, from eqs. (30) and (38), we have a family of models defined by the function $\widetilde{\omega}_{\alpha}(\tilde{h})$ and the potential $\widetilde{V}_{\alpha}(\tilde{h})$ written in polynomial form as

$$
\begin{aligned}
& \widetilde{\omega}_{\alpha}(\tilde{h})=\left|\left(2 \alpha \tilde{h} T_{2 \alpha}(\tilde{h})+U_{2 \alpha-1}(\tilde{h})\right) / 4 \alpha \tilde{h}^{3}\right|, \\
& \widetilde{V}_{\alpha}(\tilde{h})=\left(1-\tilde{h}^{2}\right)^{2} U_{2 \alpha-1}^{2}(\tilde{h}) \widetilde{\omega}_{\alpha}(\tilde{h}) / 4 \alpha^{2} .
\end{aligned}
$$

Then, each value of the parameter $\alpha$ specifies a model of this family. In particular, for $\alpha=2,3$ we have

$$
\begin{aligned}
& \widetilde{\omega}_{2}(\tilde{h})=3-4 \tilde{h}^{2}, \\
& \widetilde{V}_{2}(\tilde{h})=\tilde{h}^{2}\left(1-\tilde{h}^{2}\right)^{2}\left(1-2 \tilde{h}^{2}\right)^{2} \widetilde{\omega}_{2}(\tilde{h}), \\
& \widetilde{\omega}_{3}(\tilde{h})=\left(19-64 \tilde{h}^{2}+48 \tilde{h}^{4}\right) / 3, \\
& \widetilde{V}_{3}(\tilde{h})=h^{2}\left(1-\tilde{h}^{2}\right)^{2}\left(1-2 \tilde{h}^{2}\right)^{2}\left(3-4 \tilde{h}^{2}\right)^{2} \widetilde{\omega}_{3}(\tilde{h}) / 9 .
\end{aligned}
$$

For these models, from the inverse of the deformation function (38), we obtain the static Higgs field solutions as

$$
\tilde{h}^{( \pm)}(x)=\cos \left(\left[\arccos \left(h^{( \pm)}(x)\right)+(m-1) \pi\right] / \alpha\right),
$$

where $h^{( \pm)}(x)$ are given by eqs. (23) and (24), and $m$ is an integer, which generates distinct solutions only for $m=0, \ldots, \alpha-1$.

Let us examine the model for $\alpha=2$, defined by eqs. (44) and (45), and displayed in fig. 8. We see that the potential is positive only for $\tilde{h} \leqslant \sqrt{3} / 2$. Then, there are two kinds of static solutions for the Higgs field, one pair kink/antikink-like solution between $0 \leqslant \tilde{h} \leqslant 1 / \sqrt{2}$, and a lump-like solution between $1 / \sqrt{2} \leqslant \tilde{h} \leqslant \sqrt{3} / 2$. In ref. [21] a model that presents a charged lump-like solution was considered. Here, the lump-like solution presents vanishing charges and energy, hence we examine only the wall for $0 \leqslant \tilde{h} \leqslant$ $1 / \sqrt{2}$. In fig. 9, we display the Higgs field (48) and the gauge field (40) solutions. These walls have the same total energy and charges of the walls of the standard JLW model, but with different spacial distribution of the energy and charge densities, as shown in fig. 10. 


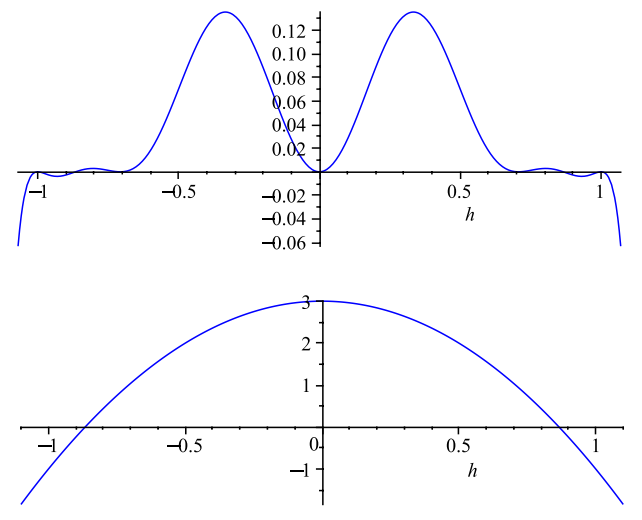

Fig. 8: (Colour on-line) The potential (45) (top panel) and the function $w$ (44) (bottom panel), as a function of $\tilde{h}$.
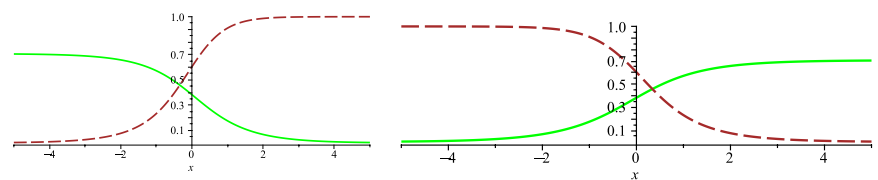

Fig. 9: (Colour on-line) The Higgs field (48) for $m=0$ and $\alpha=2$ (solid line) and the gauge field (40) (dashed line), for $\tilde{h}^{(+)}(x)$ and $\widetilde{A}^{(-)}(x)$, on the left, and for $\tilde{h}^{(-)}(x)$ and $\widetilde{A}^{(+)}(x)$, on the right.
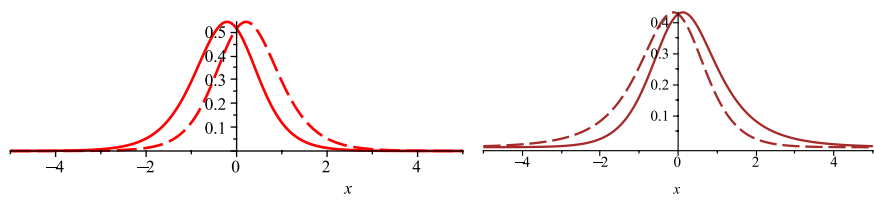

Fig. 10: (Colour on-line) The energy density (on the left) and the module of charge density (on the right), for solution $A^{(-)}(x)$ (solid line), and for $A^{(+)}(x)$ (dashed line).

Conclusion. - The examples presented above illustrate how the deformation method may be used to generate many new generalized Abelian HCS models and their analytic defect solutions. This is achieved without any need to directly solve the nonlinear equations of motion of the new models. The method also allows the construction of new defect solutions controlling important features of them such as height, width or topological character. Such results are of direct interest to applications of domain walls in several contexts, such as high-energy or condensedmatter physics.

Two of us (LL and JMCM) thank CAPES and CNPq (Brazilian agencies) for financial support. DR-G acknowledges CFP for partial support and kind hospitality. CDS is partially funded under the FCT project CERN/FP/116358/2010.

\section{REFERENCES}

[1] Rajaraman R., Solitons and Instantons (North-Holland, Amsterdam) 1982; RebBi C. and Soliani G., Solitons and Particles (World Scientific, Singapore) 1984.
[2] Vilenkin A. and Shellard E. P. S., Cosmic Strings, and Other Topological Defects (Cambridge University Press, Cambridge, UK) 1994.

[3] Eschenfelder A. H., Magnetic Bubble Technology (Springer, New York) 1997.

[4] Lerda A., Anyons: Quantum Mechanics of Particles with Fractional Statistics (Springer-Verlag, Berlin) 1992.

[5] Hagen C. R., Phys. Rev. D, 31 (1985) 848.

[6] JACKIW R. and PI S.-Y., Phys. Rev. D, 42 (1990) 3500.

[7] Bergman O. and Lozano G., Ann. Phys. (N.Y.), 229 (1994) 416.

[8] Gomes M., Malbouisson J. M. C. and Da Silva A. J., Phys. Lett. A, 236 (1997) 373; Gomes M., Malbouisson J. M. C., Rodrigues A. G. and DA Silva A. J., J. Phys. A: Math. Gen., 33 (2000) 5521.

[9] Hong J., Kim Y. and Pac P. Y., Phys. Rev. Lett., 64 (1990) 2230; Jackiw R. and Weinberg E. J., Phys. Rev. Lett., 64 (1990) 2234.

[10] Jackiw R., Lee K. and Weinberg E. J., Phys. Rev. D, 42 (1990) 3488.

[11] Bazeia D., Da Hora E., dos Santos C. and Menezes R., Phys. Rev. D, 81 (2010) 125014.

[12] dos Santos C. and DA Hora E., Eur. Phys. J. C, 70 (2010) 1145.

[13] Skyrme T. H. R., Proc. R. Soc. London, Ser. A, 260 (1961) 127; Aratyn H., Ferreira L. A. and Zimerman A. H., Phys. Rev. Lett., 83 (1999) 1723; Babichev E., Phys. Rev. D, 74 (2006) 085004; Adam C., Klimas P., Sanchez-Guillen J. and Wereszczynski A., J. Phys. A, 42 (2009) 135401.

[14] Armendariz-Picon C., Damour T. and Mukhanov V. F., Phys. Lett. B, 458 (1999) 209; Garriga J. and Mukhanov V. F., Phys. Lett. B, 458 (1999) 219; Chiba T., Okabe T. and Yamaguchi M., Phys. Rev. D, 62 (2000) 023511; VERBin Y., MADSEN S. and LARSEN A. L., Phys. Rev. D, 67 (2003) 085019; ArmendarizPicon C. and Lim E. A., JCAP, 08 (2005) 007; RENDALL A. D., Class. Quantum Grav., 23 (2006) 1557.

[15] Bazeia D., Losano L. and Malbouisson J. M. C., Phys. Rev. D, 66 (2002) 101701(R); Almeida C. A., Bazeia D., Losano L. and Malbouisson J. M. C., Phys. Rev. D, 69 (2004) 067702; Bazeia D. and Losano L., Phys. Rev. D, 73 (2006) 025016.

[16] Bazeia D., Gonzalez Leon M. A., Losano L. and Mateos Gullarte J., Phys. Rev. D, 73 (2006) 105008.

[17] Bazeia D., Losano L., Malbouisson J. M. C. and Menezes R., Physica D, 237 (2008) 937; Bazeia D., Losano L., Menezes R. and Souza M. A. M., EPL, 87 (2009) 21001; Bazeia D., Losano L., Malbouisson J. M. C. and Santos J. R. L., Eur. Phys. J. C, 71 (2011) 176.

[18] Afonso V. I., Bazeia D., Gonzalez Leon M. A., Losano L. and Mateos Gilarte J., Phys. Rev. D, 76 (2007) 025010.

[19] Bogomol'nyi E. B., Yad. Fiz., 24 (1976) 861 (Sov. J. Nucl. Phys., 24 (1976) 449); Prasad M. and SommerFELD C., Phys. Rev. Lett., 35 (1975) 760.

[20] Vega H. and Schaposnik F., Phys. Rev. D, 14 (1976) 1100.

[21] dos Santos C. and DA Hora E., Eur. J. Phys. C, 71 (2011) 1519. 